\title{
FTS and 2-DG induce pancreatic cancer cell death and tumor shrinkage in mice
}

\author{
L Goldberg ${ }^{1}, \mathrm{R}$ Israeli ${ }^{1}$ and Y Kloog ${ }^{*, 1}$
}

The Ras inhibitor S-trans-trans farnesylthiosalicylic acid (FTS) inhibits active Ras, which controls cell proliferation, differentiation, survival, and metabolism. FTS also inhibits HIF1 $\alpha$ expression in cancer cells, leading to an energy crisis. The synthetic glucose analog 2-deoxy-D-glucose (2-DG), which inhibits glycolysis, is selectively directed to tumor cells that exhibit increased glucose consumption. The 2-DG enters tumor cells, where it competes with glucose for glycolytic enzymes. In cancer models, as well as in human phase 1 trials, 2-DG inhibits tumor growth without toxicity. We postulated that under normoxic conditions, tumor cells treated with FTS would be more sensitive than normal cells to 2-DG. We show here that combined treatment with FTS and 2-DG inhibited cancer cell proliferation additively, yet induced apoptotic cell death synergistically both in vitro and in vivo. The induced apoptosis was inferred from QVD-OPH inhibition, an increase in cleaved caspase 3 , and loss of survivin. FTS and 2-DG when combined, but not separately, also induced an increase in fibrosis of the tumor tissue, chronic inflammation, and tumor shrinkage. Overall, these results suggest a possible new treatment of pancreatic tumors by the combined administration of FTS and 2-DG, which together induce pancreatic tumor cell death and tumor shrinkage under nontoxic conditions.

Cell Death and Disease (2012) 3, e284; doi:10.1038/cddis.2012.24; published online 15 March 2012

Subject Category: Cancer Metabolism

Ras proteins serve as key regulators of oncogenic processes by governing cell proliferation, migration, and survival. ${ }^{1-3}$ Inactive Ras proteins are attached to GDP and become activated when receptors, in response to extracellular signals, activate Ras guanine-nucleotide exchange factors to induce exchange of GDP for GTP. ${ }^{4,5}$ The GTP-bound active Ras may then target a multitude of downstream effectors, causing their activation, which normally is transient owing to the hydrolysis of GTP facilitated by Ras GTPase-activating proteins (RasGAPs). ${ }^{6-8}$ However, specific mutations at codons 12 and 13 render Ras insensitive to Ras-GAPs, and a mutation at codon 61 eliminates the intrinsic GTPase catalytic activity of the Ras protein. ${ }^{1}$ A large percentage of all cancer types either express one of the mutationally activated Ras isoforms or harbor a chronically activated Ras isoform owing to overexpression of growth-factor receptors. ${ }^{9,10}$ Therefore, Ras has long been considered an appropriate potential target for directed therapy, but attempts to target Ras with agents, such as farnesyltransferase or methyltransferase inhibitors, have so far been unsuccessful. ${ }^{1}$

Over the last 15 years, we have elaborated a new concept and developed a new class of Ras inhibitors that specifically affect the active form of Ras. The concept is based on the knowledge that the farnesyl moiety common to all Ras proteins ${ }^{11,12}$ not only serves as a lipophilic lipid anchor, but also confers functional specificity on Ras. ${ }^{1,11,12}$ Those findings raised the possibility that the farnesyl group might act as part of a recognition unit for specific anchorage lipids or protein(s) that interact with Ras in the cell membrane. Such Ras-binding partners were identified when it was shown that galectin-1 and galectin- $3,{ }^{13}$ respectively, act as specific binding partners only of farnesylated active $\mathrm{H}$-Ras and K-Ras. ${ }^{14-16}$ Hydrophobic binding pockets in these galectins are the putative farnesyl-binding sites. ${ }^{16,17}$ Galectin-1 drives the formation and participates as an integral component of $\mathrm{H}$-Ras-GTP nanoclusters, the sites at which Raf is activated. $^{18}$ Galectin-3 drives K-Ras-GTP nanoclustering, leading to robust Ras signaling. ${ }^{19}$ Interaction of nucleolin with $\mathrm{N}$-Ras and the epidermal growth factor receptor was recently shown to synergistically support N-Ras transformation in vitro. $^{20}$ Taken together, and assuming that compounds that block farnesyl-binding sites would act as Ras inhibitors, these findings point to the farnesyl-binding pockets as excellent potential targets for Ras-directed therapy. The original design of the specific synthetic Ras inhibitor, S-trans-trans farnesylthiosalicylic acid (FTS; salirasib), was indeed based on that assumption. FTS, which was designed to mimic the farnesyl moiety in the carboxy terminal of Ras, was found to induce accelerated dislodgement of Ras from the cell membrane and its subsequent degradation. ${ }^{21}$ FTS also inhibits tumor growth, cell migration, and energy metabolism in numerous cancer cell lines and many types of cancer cells that exhibit high Ras GTP levels. ${ }^{22-26}$ The most recent clinical trials showed that oral salirasib treatment of patients with pancreatic cancer increases survival rates (http://www.concordiapharma.com).

\footnotetext{
'Department of Neurobiology, George S Wise Faculty of Life Sciences, Tel Aviv University, 69978 Tel Aviv, Israel

*Corresponding author: Y Kloog, Department of Neurobiology, George S Wise Faculty of Life Sciences, Tel Aviv University, 69978 Tel Aviv, Israel. Tel: + 97234966440 ;

Fax: + 9723640 7643; E-mail: kloog@post.tau.ac.il

Keywords: Ras; Ras-oncogenes; 2-DG; FTS; pancreatic cancer

Abbreviations: Ras-GAPs, Ras GTPase activating proteins; 2-DG, 2-deoxy glucose; FTS, salirasib, S-trans-trans farnesylthiosalicylic acid

Received 13.2.12; accepted 14.2.12; Edited by G Melino
} 
Biochemical and gene-expression profiling experiments strongly support the notion that the tumor growth inhibitory effects of FTS result from inhibition of the active Ras and the Ras-dependent signaling that participate in tumor progression and maintenance. ${ }^{22-26}$ An interesting additional outcome of FTS treatment in tumor cells is energy crisis. ${ }^{27}$ Malignant cells, even under aerobic conditions, rely mostly on glycolysis for their energy supply. In glioblastoma cells, for example, by blocking the expression of HIF1 $\alpha$ and hence the expression of various enzymes of the glycolysis cycle, FTS causes a severe energy deficit, leading eventually to cell death. ${ }^{27}$ This finding provided the basis for a novel strategy in which FTS is combined with compounds that block glycolysis by mechanisms other than blocking of HIF $1 \alpha$ expression. The idea is to use inhibitors of energy pathways that act independently of the inhibitory activity of FTS, but inhibit enzymes that are essential for tumor cell proliferation and survival.

The well-known metabolic inhibitor 2-deoxy-D-glucose (2-DG) is a synthetic glucose analog that is selectively directed to tumor cells, which, under hypoxic conditions, exhibit increased glucose consumption facilitated by glucose transporters. ${ }^{28,29}$ Following its active transport into the tumor cells, 2-DG competes with glucose for key enzymes in glycolysis. ${ }^{28,29}$ The 2-DG has also been shown to exhibit anti-tumor activity in mouse and rat cancer models in vivo, including models of sarcomas, adenocarcinomas, leukemias, melanomas, and bladder, colon, lung, and breast tumors. ${ }^{30-32}$ Clinical trials aimed at evaluating the hormonal and metabolic effects of glucose deprivation have shown that 2-DG, although known to be toxic at high doses, does not cause any serious adverse events at doses of up to $200 \mathrm{mg} / \mathrm{kg}$ (a single intravenous dose). In addition, when evaluated in phase 1 clinical trials of patients suffering from recurrent solid tumors, 2-DG was found to be safe and to exhibit some antitumor effects (www.thresholdpharm.com).

We postulated that tumor cells treated with FTS would be highly sensitive to metabolic inhibitors, probably significantly more sensitive than normal cells under normoxic conditions. Here we demonstrate that the combination of FTS and 2-DG synergistically attenuated pancreatic cancer cell proliferation in vitro and caused significant shrinkage of pancreatic tumors in mice. Furthermore, this combined treatment, both in vitro and in vivo, synergistically induced apoptotic death of tumor cells, which was not induced by FTS alone.

\section{Results}

Combined treatment with FTS and 2-DG synergistically induces cell death of panc-1 pancreatic carcinoma cells. FTS has been shown to inhibit the anchoragedependent and -independent proliferation of panc-1 and MIA PaCa-2 pancreatic cell lines that harbor oncogenic K-Ras, without inducing cell death. ${ }^{24,26}$ The first set of experiments with 2-DG in the present study was aimed at establishing a dosage at which the effect of 2-DG on cell survival, when administered by itself, is minimal. Increasing the ratio of 2-DG to glucose has been shown to result in tumor cell death. ${ }^{33}$ In this study, therefore, we employed a non-toxic dose, $5 \mathrm{mM}$ 2-DG, combined with equimolar glucose, which by itself is not toxic and has been shown, for example, to enhance the cytotoxicity of topoisomerase inhibitors. ${ }^{34}$ The extent of death of 2-DG-treated panc-1 cells in our experiments was small and was similar to that observed in untreated controls ( $3 \%$ death), confirming that 2-DG alone, at least at the dosage employed here, does not affect panc-1 cell survival. We therefore used this non-toxic dose of 2-DG for all subsequent experiments.

Next, we performed a four-armed experiment in which panc- 1 cells were treated with the vehicle (DMSO) only, 2-DG alone, FTS alone, and FTS combined with 2-DG (FTS + 2-DG), as described in Materials and Methods. Images from a typical experiment performed with panc- 1 cells are shown in Figure 1a. Phase-contrast images show that treatment with FTS alone $(75 \mu \mathrm{M})$ induced, as expected, a decrease in cell number (by about $50 \%$; Figures $1 \mathrm{a}$ and $\mathrm{b}$ ). Treatment with 2-DG alone ( $5 \mathrm{mM}$ ) also resulted in a decrease in cell number, but the reduction was smaller (20-25\%, Figure 1b). Hoechst staining showed that neither of these single-drug treatments caused a significant increase in cell death, compared with the control (Figures 1a and c); the percentages of dead cells in the control (DMSO and $10 \mathrm{mM}$ glucose) and in cells treated with 2-DG (5 mM 2-DG $+5 \mathrm{mM}$ glucose), or FTS $(50 \mu \mathrm{M}$ FTS $+10 \mathrm{mM}$ glucose or $75 \mu \mathrm{M}$ FTS $+10 \mathrm{mM}$ glucose) were $1.95 \pm 1.4,2.5 \pm 4.5,3.3 \pm 1.2$, and $7.4 \pm 5.7$, respectively (Figure 1c). In contrast, combined treatment with FTS + 2-DG caused a significant increase in cell death; in cells treated with FTS $(50 \mu \mathrm{M})+2-\mathrm{DG}$, and with FTS $(75 \mu \mathrm{M})+2-\mathrm{DG}$, the percentages of dead cells were $11.5 \pm 5.8$ and $30 \pm 9.3$, respectively (Figure 1c). Thus, under conditions in which neither FTS nor 2-DG alone induced death of panc-1 cells, significant cell death was induced by their combination. This result clearly points to a synergistic effect of the two drugs in killing the panc- 1 carcinoma cells.

Combined treatment with FTS and 2-DG induces cell death in primary pancreatic carcinoma cell lines. We then performed a similar set of experiments to determine whether the effects of treatment with FTS and with 2-DG observed in panc- 1 cells could also be obtained in primary pancreatic tumor cells, which better mimic the actual disease. The cells used for this purpose were from the recently characterized human primary pancreatic carcinoma cell lines pp161, pp78, and pp109. ${ }^{35}$ All three cell lines possess an oncogenic K-Ras allele, and pp161 and pp78, but not pp109, also bear a p53 loss-of-function mutation. Hoechst staining showed that relative to the control, neither of the single-drug treatments caused significant cell death in pp78 or pp161 cells (Figures 2a and c), but both induced significant death in pp109 cells (Figure 2b). However, the combined treatment with $75 \mu \mathrm{M}$ FTS $+2-\mathrm{DG}$ significantly increased cell death in all three cell lines, as in panc-1 cells. The phase contrast images show that $75 \mu \mathrm{M}$ FTS alone induced a decrease of about $50 \%$ in the total numbers of $\mathrm{pp}$ 109 (Figure 2e), pp161 (Figure 2f), and pp 78 cells (Figure 2d). Treatment with 2-DG alone also resulted in a decrease in cell number, but its effect was weaker than that of FTS alone (Figures 2d-f). Interestingly, in pp78 and pp161, both mutant p53 cell lines, the combined treatment 

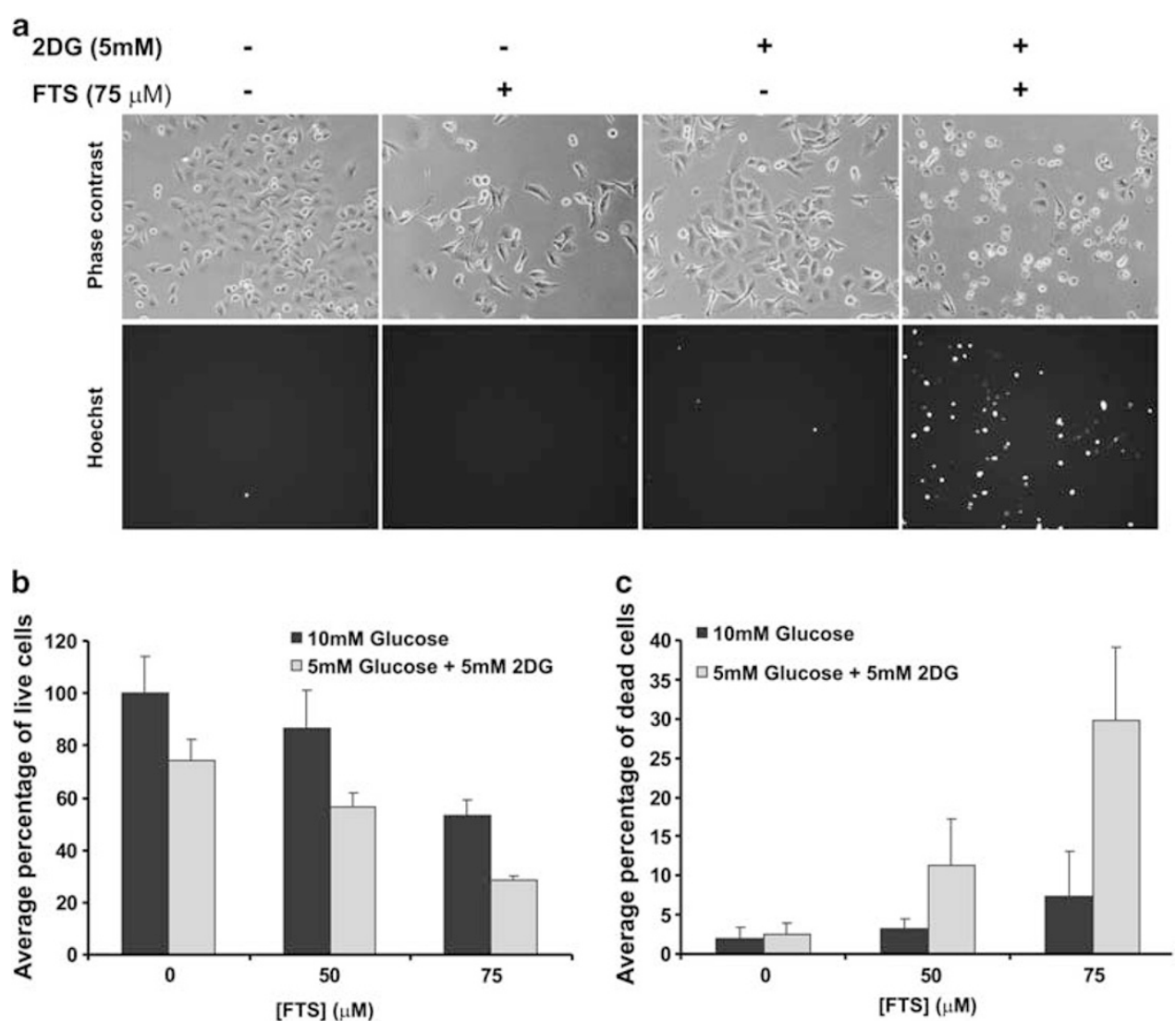

Figure 1 Combined treatment with FTS and 2-DG induces death of panc-1 cells. The panc-1 cells were seeded at a density of 10000 cells per well in $24-w e l l$ plates. After $24 \mathrm{~h}$, the media were replaced with media containing $5 \% \mathrm{FCS}$, and the cells were treated with $0.1 \%$ DMSO (control) or with FTS at the indicated concentrations. After $48 \mathrm{~h}$, the media were again replaced with media containing $5 \% \mathrm{FCS}$, and the cells were treated with $10 \mathrm{mM}$ glucose or with $5 \mathrm{mM}$ glucose and $5 \mathrm{mM}$ 2-DG, and FTS was replenished. After an additional $24 \mathrm{~h}$, the cells were stained with Hoechst and imaged using an Olympus fluorescence microscope. Typical phase-contrast (upper panels) and Hoechst fluorescent images (lower panels) are shown in (a). The number of live cells, expressed as a percentage of the number of glucose-treated control cells (mean \pm S.D.), is presented in (b). Cell death (mean \pm S.D.) was calculated as the number of Hoechst-positive cells counted in fluorescence images expressed as a percentage of the total number of cells counted in phase-contrast images (c). The experiments were performed in quadruplicate and repeated twice $(n=2)$

exerted a synergistic effect, whereas in the wild-type p53 cell line pp109, the effect was additive. The reason for this phenomenon is not yet known. However, a beneficial effect of FTS + 2-DG on cell death was observed in all three primary pancreatic carcinoma cells, as in panc- 1 cells.

\section{Combined treatment with FTS and 2-DG induces caspase-dependent apoptosis in panc-1 cells.} Consistently with early reports, FTS $(75 \mu \mathrm{M})$ alone and $5 \mathrm{mM}$ 2-DG alone, both reduced the amount of Ras in the cells, and a similar reduction was achieved with their combined treatment (Figure 3a). To determine whether the cell death induced by FTS +2-DG in panc- 1 cells was apoptotic in nature, we used the pan-caspase inhibitor QVD$\mathrm{OPH},{ }^{36}$ which inhibits the formation of cleaved (active) caspase 3 (Figure $3 a$ ). We found that death induced by the combination of FTS and 2-DG was significantly inhibited by QVD-OPH (Figure 3b), suggesting that the cell death induced by FTS + 2-DG was, at least in part, apoptotic in nature and dependent on caspase activation. This was confirmed by the observed increase in active (cleaved) caspase 3 by FTS but not 2-DG, and by marked enhancement of the cleaved caspase 3 by FTS + 2-DG, apparently similar to that observed with staurosporine, a wellknown inducer of apoptosis (Figure 3a).

In previous studies, we found that FTS induces a marked decrease in the apoptosis inhibitor survivin. ${ }^{37}$ We therefore examined the effect of FTS + 2-DG on survivin levels in panc- 1 cells. Although treatment with each drug on its own decreased survivin levels to some extent, the combined treatment completely eliminated the protein in a synergistic manner (Figure 3a). This effect was even stronger than that induced by staurosporine (Figure 3a). It is important to note that treatment with FTS + 2-DG can block the main energy source of cancer cells, both by reducing their expression of HIF $1 \alpha$ and by competing with glucose for glycolysis. ${ }^{27}$ This dual attack on energy metabolism, together with other anticancer effects of FTS (such as attenuation of cell-cycle progression ${ }^{38}$ ), may lead to the catastrophic cellular stress terminating in apoptotic cell death, as observed here.

Treatment with FTS+2-DG synergistically induces shrinkage of panc-1 tumors in nude mice. To examine the effect of treatment with FTS + 2-DG in vivo, we implanted 

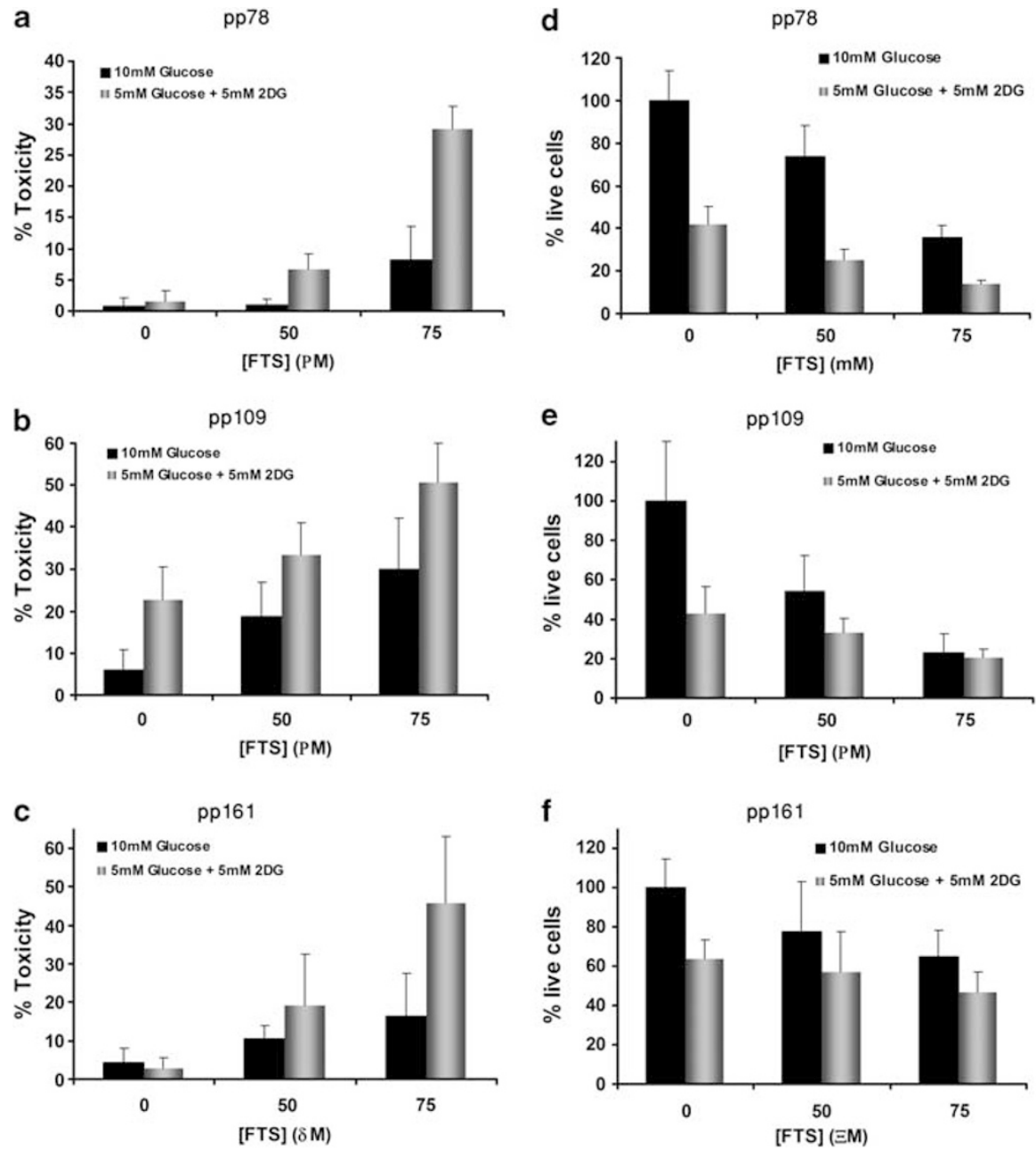

Figure 2 Combined treatment with FTS and 2-DG induces cell death of primary pancreatic tumor cells. The three primary pancreatic tumor cell lines, pp78, pp109, and pp161 were treated with FTS, 2-DG, or FTS + 2-DG as described in Figure 1 for panc-1 cells. Cell death, namely toxicity, (average \pm S.D.), calculated as in Figure 1c as the number of Hoechst-positive cells expressed as a percentage of the total number of cells, is presented in (a), (b), and (c) for pp78, pp109, and pp161, respectively. The percentage of live cells (mean \pm S.D.) relative to the untreated control is presented in (d), (e), and (f) for pp78, pp109, and pp161, respectively. The experiments were performed in quadruplicate and repeated twice $(n=2)$

panc- 1 cells subcutaneously just above the right femoral joint of nude mice. Once palpable tumors were observed, oral drug treatment was started (see Materials and Methods). After 18 days, tumors were removed and weighed. As shown in Figure 4, the average tumor weight in the group treated with 2-DG $(1000 \mathrm{mg} / \mathrm{kg})$ did not differ significantly from that of the vehicle-treated control group. Tumors treated with FTS $(60 \mathrm{mg} / \mathrm{kg})$, consistently with our previous findings, weighed $50 \%$ less, on average, than tumors excised from the vehicletreated controls. ${ }^{24}$ Remarkably, however, treatment with FTS + 2-DG resulted in tumors that weighed $90 \%$ less, on average, than the controls, with no toxic side effects. Some mice even exhibited tumor remission (data not shown). These results clearly point to a synergistic effect of $\mathrm{FTS}+2-\mathrm{DG}$ in inhibiting tumor growth, similar to the synergism observed in vitro.

Typical H\&E sections from tumors removed from the mice for pathological analysis, as described in Materials and Methods and shown in Figure 5, revealed the following significant changes in tumor morphology in the group treated with FTS + 2-DG. First, in contrast to the similarity in invasiveness between tumors from the control group and from the groups treated by the single drugs, tumors treated with FTS + 2-DG appeared much less invasive, and their edges were not as irregular. Second, tumors treated with either of the single drugs exhibited only slightly more fibrosis than the controls, especially at their edges, whereas tumors treated with FTS + 2-DG exhibited marked fibrosis around the tumor edges and at the leading edges (Figure 5, white arrow in hematoxylin and eosin (H\&E)). Third, only the tumors treated with FTS + 2-DG exhibited significant chronic inflammation (Figure 5, yellow arrow in H\&E) and fibrosis.

These changes in tumor morphology suggest that treatment with FTS + 2-DG induced death of the tumor cells in the tissue. To verify this notion, we stained tumor sections with TUNEL to identify apoptotic cell death. As shown in Figure 5, tumors treated with either of the single drugs showed some TUNEL staining, which was altogether lacking in the control 
a
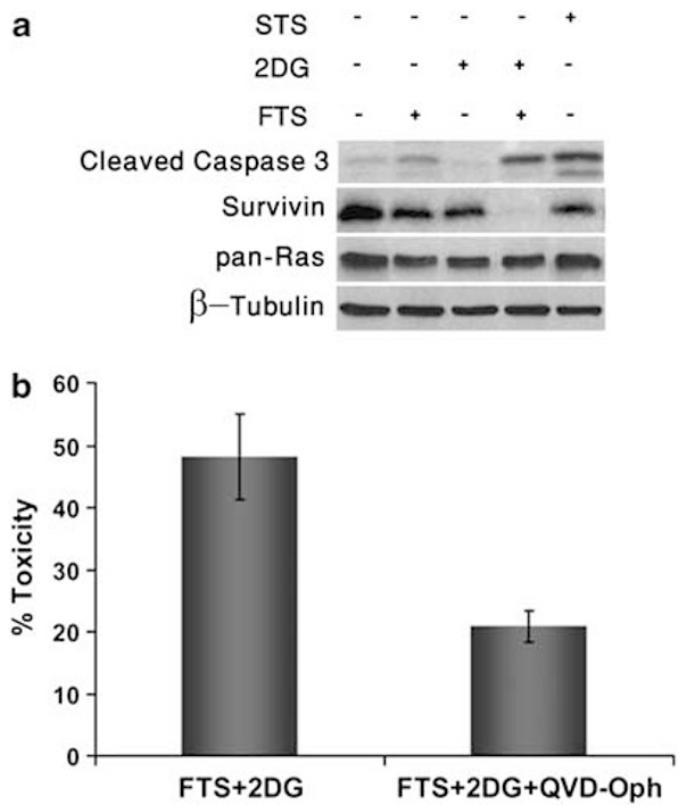

Figure 3 Combined treatment with FTS and 2-DG induces caspase activation in panc-1 cells. (a) The panc- 1 cells were seeded at a density of 10000 cells per well in six-well plates. After $24 \mathrm{~h}$, the media were replaced with media containing $5 \%$ FCS, and the cells were treated with the vehicle (DMSO $0.1 \%$; control) or with staurosporine (STS, positive control), $5 \mathrm{mM}$ 2-DG, $75 \mu \mathrm{M}$ FTS, or FTS + 2-DG. After $48 \mathrm{~h}$, the cells were lysed and the lysates were subjected to immunoblotting with antibodies to cleaved caspase 3 , survivin, pan-Ras, and $\beta$-tubulin (loading control), as described earlier. The experiment was repeated twice $(n=2)$ with similar results that show a marked increase in cleaved caspase 3 and decrease in survivin in the combination of 2-DG and FTS treatment. (b) Induction of panc-1 cell death by FTS + 2-DG is blocked by a caspase inhibitor. The panc-1 cells were seeded at a density of 10000 cells per well in 24-well plates, as described for Figure $3 \mathrm{a}$. After $24 \mathrm{~h}$, the media were replaced with media containing $5 \% \mathrm{FCS}$, and the cells were treated with DMSO $(0.1 \%)$ or with FTS $(75 \mu \mathrm{M})$. After $48 \mathrm{~h}$, the media were again replaced with media containing $5 \% \mathrm{FCS}, 10 \mathrm{mM}$ glucose, or with $5 \mathrm{mM}$ glucose and $5 \mathrm{mM}$ 2-DG, and FTS was replenished. The caspase inhibitor QVD$\mathrm{OPh}(20 \mu \mathrm{M})$ was then added to the cells treated with FTS $+2-\mathrm{DG}$. After an additional $48 \mathrm{~h}$, the cells were stained with Hoechst, imaged using an Olympus fluorescence microscope, and the percent cell death (toxicity) was calculated as described in Figure $1 b$. Cell death (mean \pm S.D.) was calculated as the number of Hoechst-stained cells counted in fluorescence images, expressed as a percentage of the total number of cells counted in phase-contrast images (right panel). The experiment was performed in quadruplicate and repeated $(n=2)$

tumors. However, tumors treated with FTS + 2-DG exhibited strong and significant TUNEL staining, especially at the tumor edges, indicating massive apoptotic cell death of the tumor tissue (Figure 5). These findings confirmed that treatment of panc-1 tumors with FTS + 2-DG in mice significantly inhibited tumor growth by inducing apoptosis of the tumor cells.

\section{Discussion}

Activating mutations in Ras contribute to $30 \%$ of all human tumors, ${ }^{1}$ suggesting Ras as a prime target for cancer therapy. The specific Ras inhibitor, FTS, successfully competes with Ras chaperones for binding to active Ras, rendering it inactive and thus causing inhibition of tumor cell proliferation in vitro and in vivo. ${ }^{14,15,20}$ In a recent study, it was shown, moreover, that 2-DG induced HIF $1 \alpha$-independent apoptosis in rhabdomyosarcoma cells. ${ }^{39}$

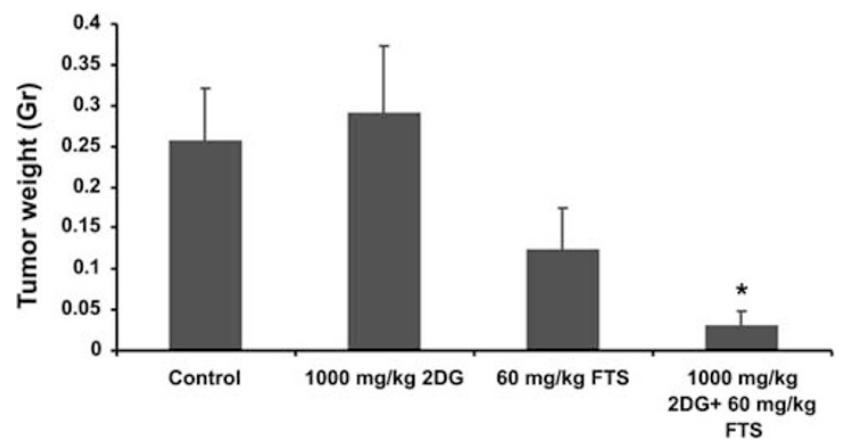

Figure 4 Combined treatment with FTS + 2-DG synergistically inhibits growth of panc-1 tumors in nude mice. Nude $\mathrm{CD}_{1}$-Nu mice were xenografted with $7 \times 10^{6}$ panc-1 cells (subcutaneously above the right femoral joint). After 10 days, the mice were separated into four groups: three experimental groups that received daily oral doses of FTS $(60 \mathrm{mg} / \mathrm{kg}), 2-D G(1000 \mathrm{mg} / \mathrm{kg}), F T S+2-D G$, or vehicle $(0.5 \%$ carboxymethylcellulose). Tumors were extracted and weighed after 23 days. Typical results (mean \pm S.D., $n=7$; ${ }^{*} P<0.05$, Kruskal-Wallis test) obtained from one of the two $(n=2)$ experiments performed are presented

In the present study, both FTS and 2-DG, when administered as single agents, were found to inhibit the proliferation of panc-1 tumor cells, and when administered in combination, their effect on such tumor growth was additive. In contrast, whereas monotherapy with FTS or with a low (non-toxic) dose of the glycolysis inhibitor 2-DG was incapable of causing significant death in this pancreatic tumor cell line, combined administration of the two agents prompted their synergistic effect resulting in significant cell death, as demonstrated in vitro by Hoechst labeling and in vivo by TUNEL. A beneficial effect of FTS + 2-DG on cell death was also obtained in three human primary pancreatic carcinoma cell lines ${ }^{35}$ (additively in the pp109 cell line and synergistically in pp78 and pp161). Proof that this cell death was apoptotic in nature came from its observed inhibition by the apoptosis inhibitor QD-OPH (Figure $3 \mathrm{~b}$ ), as well as from the increase in active (cleaved) caspase 3 and decrease in pro-caspases 7 and 9 (not shown) induced by FTS + 2-DG. Further evidence came from the fact that the apoptosis inhibitor survivin, in which FTS by itself induces a marked decrease, ${ }^{37}$ was completely eliminated in response to the combined treatment (Figure 3a).

It is important to point out that FTS itself is not cytotoxic either in vitro or in vivo. Our in-vivo experiments in mice, in which tumors induced by implantation of panc- 1 cells were treated with FTS combined with non-toxic dose of 2-DG, resulted in shrinkage of pancreatic tumors and changes in tumor morphology as described in detail in Results - is an unexpected and novel finding.

Taken together, our results suggest a possible novel treatment of pancreatic tumor by combining FTS and 2-DG under non-toxic conditions. This can be expected to provide synergistic tumor shrinkage, cell death, and possibly, also remission.

\section{Materials and Methods}

Cell culture and immunoblotting. The human pancreatic cancer cell line panc-1 was obtained from the American Tissue Culture Association. Human primary pancreatic cancer cells pp78, pp109, and pp161 were kindly donated by Andrea Cavazzana (University of Pisa, Pisa). All cells were maintained in DMEM/ $10 \%$ fetal 


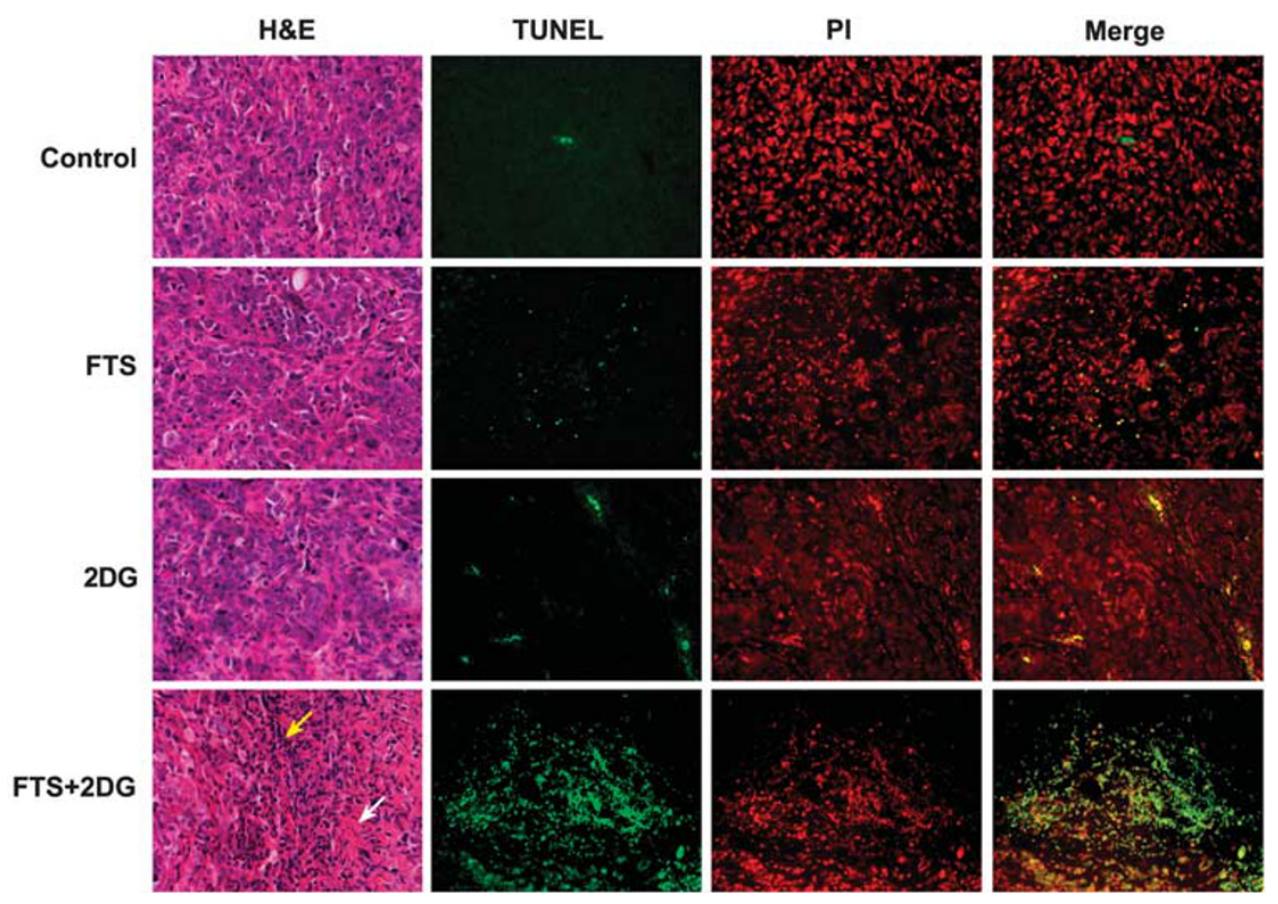

Figure 5 FTS and 2-DG induce cell death of panc-1 tumors in nude mice. The panc-1 tumors excised from untreated (control) or Nude $\mathrm{CD}_{1}$-Nu mice treated with FTS or 2-DG, or FTS + 2-DG were sliced to 10 and 5- $\mu \mathrm{m}$ sections, and prepared for histology as described in Materials and Methods. The 10- $\mu \mathrm{m}$ sections were stained with H\&E and imaged using an Olympus fluorescent microscope (original magnification $\times 200$ ). The $5-\mu \mathrm{m}$ sections were stained by TUNEL or propidium iodide (PI) according to the manufacturer's instructions, and the stained sections were imaged using a Nikon fluorescence microscope (original magnification $\times 200$ ). The white arrow in the H\&E-stained section indicates an area of fibrosis. The yellow arrow indicates infiltration of immune cells. Typical results obtained from one of the two $(n=7)$ experiments performed are presented

calf serum (FCS) at a constant temperature of $37^{\circ} \mathrm{C}$ in a humidified atmosphere of $95 \%$ air $/ 5 \% \mathrm{CO}_{2}$. Immunoblotting of Ras, cleaved caspase 3, survivin and betatubulin were performed with specific Ab as previously described. ${ }^{27}$ Materials used for cell culture and determination of cell proliferation and death were all as previously described. ${ }^{27}$ FTS (batch number 012894-A-01-003) was provided by Concordia Pharmaceuticals (Fort Lauderdale, FL, USA). 2-DG was purchased from Sigma (St. Louis, MO, USA; cat number D3179).

Determination of cell proliferation and death. Cells (panc- 1 or human primary pancreatic tumor cells) were plated in $1 \mathrm{ml}$ of DMEM containing $5 \% \mathrm{FCS}$ in 24-well plates at a density of 10000 per well and treated $24 \mathrm{~h}$ later with FTS (50 or $75 \mu \mathrm{M})$ or vehicle ( $0.1 \% \mathrm{DMSO})$. After an additional $48 \mathrm{~h}$, the media were replaced by medium containing either $10 \mathrm{mM}$ glucose, or $5 \mathrm{mM}$ glucose and $5 \mathrm{mM}$ 2-DG. FTS-treated cells were replenished with FTS or FTS plus 2-DG, or glucose with $0.1 \%$ DMSO (control). After incubation for 24 or $48 \mathrm{~h}$, as specified for each experiment, the extent of cell death was determined by staining of the cells for $5 \mathrm{~min}$ with Hoechst $33258(1 \mu \mathrm{g} / \mathrm{ml})$. Stained nuclei (dead cells) were visualized under a fluorescence microscope. Phase-contrast images were also obtained. All experiments were performed in quadruplicate and were repeated four times. Data were collected by imaging of each well. The total number of cells was obtained by counting them in the phase-contrast images. The number of dead cells were counted from the fluorescence images and expressed as percentages (means \pm S.D.) of the total number of cells.

Tumor implantation in mice. Nude $C D_{1}$-Nu mice ( 8 weeks old) were housed in barrier facilities on a 12-h light/dark cycle. Food and water were supplied ad libitum. On day $0,7 \times 10^{6}$ panc- 1 cells in $0.1 \mathrm{ml}$ of phosphate-buffered saline were xenografted subcutaneously just above the right femoral joint.

On day 10, tumors were measured and the mice were divided into four groups for treatment with daily oral doses $(300 \mu \mathrm{l})$ of FTS $(60 \mathrm{mg} / \mathrm{kg})$ and $/$ or 2-DG $(1000 \mathrm{mg} / \mathrm{kg}$ ), or of $0.5 \%$ carboxymethylcellulose (control).

After 23 days of treatment, tumors were extracted and weighed. The experiment was performed twice, and average tumor weights for each group were calculated (means \pm S.D., $n=7$ per experiment). Statistical significance of the results was determined by non-parametric ANOVA (Kruskal-Wallis test).

Histology and TUNEL staining. Panc-1 tumors were extracted and histological sections (5 and $10 \mu \mathrm{m})$ were prepared. The 10- $\mu \mathrm{m}$ sections were stained with $\mathrm{H} \& \mathrm{E}$ and were then imaged using an Olympus fluorescence microscope (Olympus Europa Holding GmbH, Hamburg, Germany) (original magnification $\times 200$ ). H\&E sections were also sent for thorough pathological examination. The $5-\mu \mathrm{m}$ sections were subjected to TUNEL according to the manufacturer's instructions (MBL International, Woburn, MA, USA) and were then imaged using a Nikon fluorescence microscope (Nikon Instech, Tokyo, Japan) (original magnification $\times 200$ ).

\section{Conflict of Interest}

The authors declare no conflict of interest.

Acknowledgements. This work was supported in part by The Israel Science Foundation (YK), the Prajs-Drimmer Institute for the Development of AntiDegenerative Drugs (LG), and the SFAHO Foundation (a private Foundation; YK). $Y$ Kloog is the incumbent of the Jack $\mathrm{H}$ Skirball Chair in Applied Neurobiology and Head of the Prajs-Drimmer Institute.

1. Cox AD, Der CJ. Ras history: the saga continues. Small Gtpases 2011; 1: 2-27.

2. Downward J. Ras signalling and apoptosis. Curr Opin Genet Dev 1998; 8: 49-54.

3. Joneson T, White MA, Wigler MH, Bar Sagi D. Stimulation of membrane ruffling and MAP kinase activation by distinct effectors of RAS. Science 1996; 271: 810-812.

4. Campbell SL, Khosravi-Far R, Rossman KL, Clark GJ, Der CJ. Increasing complexity of Ras signaling. Oncogene 1998; 17 (11 Reviews): 1395-1413.

5. McCormick F. Signal transduction. How receptors turn Ras on. Nature 1993; 363: 15-16. 
6. Boguski MS, McCormick F. Proteins regulating Ras and its relatives. Nature 1993; 366 643-654.

7. Scheffzek K, Ahmadian MR, Kabsch W, Wiesmuller L, Lautwein A, Schmitz F et al. The Ras-RasGAP complex: structural basis for GTPase activation and its loss in oncogenic Ras mutants. Science 1997; 277: 333-338.

8. Wittinghofer A, Vetter IR. Structure-function relationships of the $G$ domain, a canonical switch motif. Annu Rev Biochem 2011; 80: 943-971.

9. Egan SE, Giddings BW, Brooks MW, Buday L, Sizeland AM, Weinberg RA. Association of Sos Ras exchange protein with Grb2 is implicated in tyrosine kinase signal transduction and transformation. Nature 1993; 363: 45-51.

10. Gale NW, Kaplan S, Lowenstein EJ, Schlessinger J, Bar-Sagi D. Grb2 mediates the EGFdependent activation of guanine nucleotide exchange on Ras. Nature 1993; 363: 88-92.

11. Boyartchuk VL, Ashby MN, Rine J. Modulation of Ras and a-factor function by carboxylterminal proteolysis. Science 1997; 275: 1796-1800.

12. Hancock JF, Cadwallader K, Marshall CJ. Methylation and proteolysis are essential for efficient membrane binding of prenylated p21K-ras(B). EMBO J 1991; 10: 641-646.

13. Shoji H, Deltour L, Nakamura T, Tajbakhsh S, Poirier F. Expression pattern and role of Galectin1 during early mouse myogenesis. Dev Growth Differ 2009; 51: 607-615.

14. Elad-Sfadia G, Haklai R, Balan E, Kloog Y. Galectin-3 augments K-Ras activation and triggers a Ras signal that attenuates ERK but not phosphoinositide 3-kinase activity. J Bio Chem 2004; 279: 34922-34930.

15. Elad-Sfadia G, Haklai R, Ballan E, Gabius HJ, Kloog Y. Galectin-1 augments Ras activation and diverts Ras signals to Raf-1 at the expense of phosphoinositide 3-kinase. J Biol Chem 2002; 277: 37169-37175.

16. Shalom-Feuerstein R, Cooks T, Raz A, Kloog Y. Galectin-3 regulates a molecular switch from N-Ras to K-Ras usage in human breast carcinoma cells. Cancer Res 2005; 65 . 7292-7300.

17. Rotblat B, Niv H, Andre S, Kaltner H, Gabius HJ, Kloog Y. Galectin-1(L11A) predicted from a computed galectin-1 farnesyl-binding pocket selectively inhibits Ras-GTP. Cancer Res 2004; 64: 3112-3118.

18. Rotblat B, Belanis L, Liang H, Haklai R, Elad-Zefadia G, Hancock JF et al. H-Ras nanocluster stability regulates the magnitude of MAPK signal output. PLoS One 2010; 5 : e11991.

19. Shalom-Feuerstein R, Plowman SJ, Rotblat B, Ariotti N, Tian T, Hancock JF et al. K-ras nanoclustering is subverted by overexpression of the scaffold protein galectin-3. Cancer Res 2008; 68: 6608-6616.

20. Farin K, Schokoroy S, Haklai R, Cohen-Or I, Elad-Sfadia G, Reyes-Reyes ME et al Oncogenic synergism between ErbB1, nucleolin, and mutant Ras. Cancer Res 2011; 71 2140-2151.

21. Haklai R, Gana-Weisz G, Elad G, Paz A, Marciano D, Egozi Y et al. Dislodgment and accelerated degradation of Ras. Biochemistry 1998; 37: 1306-1314.

22. Elad G, Paz A, Haklai R, Marciano D, Cox A, Kloog Y. Targeting of K-Ras 4B by S-trans, trans-farnesyl thiosalicylic acid. Biochim Biophys Acta 1999; 1452: 228-242.

23. Goldberg L, Ocherashvilli A, Daniels D, Last D, Cohen ZR, Tamar G et al. Salirasib (farnesyl thiosalicylic acid) for brain tumor treatment: a convection-enhanced drug delivery study in rats. Mol Cancer Ther 2008; 7: 3609-3616.

24. Haklai R, Elad-Sfadia G, Egozi Y, Kloog Y. Orally administered FTS (Salirasib) inhibits human pancreatic tumor growth in nude mice. Cancer Chemother Pharmacol 2008; 61: 89-96.

25. Levy R, Grafi-Cohen M, Kraiem Z, Kloog Y. Galectin-3 promotes chronic activation of KRas and differentiation block in malignant thyroid carcinomas. Mol Cancer Ther 2011; 9 2208-2219.
26. Weisz B, Giehl K, Gana-Weisz M, Egozi Y, Ben-Baruch G, Marciano D et al. A new functional Ras antagonist inhibits human pancreatic tumor growth in nude mice. Oncogene 1999; 18: 2579-2588.

27. Blum R, Jacob-Hirsch J, Amariglio N, Rechavi G, Kloog Y. Ras inhibition in glioblastoma down-regulates hypoxia-inducible factor-1alpha, causing glycolysis shutdown and cell death. Cancer Res 2005; 65: 999-1006.

28. Dearling JL, Flynn AA, Sutcliffe-Goulden J, Petrie IA, Boden R, Green AJ et al. Analysis of the regional uptake of radiolabeled deoxyglucose analogs in human tumor xenografts. J Nucl Med 2004; 45: 101-107.

29. Maher JC, Krishan A, Lampidis TJ. Greater cell cycle inhibition and cytotoxicity induced by 2-deoxy-D-glucose in tumor cells treated under hypoxic versus aerobic conditions. Cancer Chemother Pharmacol 2004; 53: 116-122.

30. Aft RL, Lewis JS, Zhang F, Kim J, Welch MJ. Enhancing targeted radiotherapy by copper(II)diacetyl- bis(N4-methylthiosemicarbazone) using 2-deoxy-D-glucose. Cancer Res 2003; 63: 5496-5504.

31. Coleman MC, Asbury CR, Daniels D, Du J, Aykin-Burns N, Smith BJ et al. 2-deoxy-Dglucose causes cytotoxicity, oxidative stress, and radiosensitization in pancreatic cancer. Free Radic Biol Med 2008; 44: 322-331.

32. Maschek G, Savaraj N, Priebe W, Braunschweiger P, Hamilton K, Tidmarsh GF et al. 2-deoxy-D-glucose increases the efficacy of adriamycin and paclitaxel in human osteosarcoma and non-small cell lung cancers in vivo. Cancer Res 2004; 64: 31-34.

33. Aft RL, Zhang FW, Gius D. Evaluation of 2-deoxy-D-glucose as a chemotherapeutic agent: mechanism of cell death. Br J Cancer 2002; 87: 805-812.

34. Gupta S, Mathur R, Dwarakanath BS. The glycolytic inhibitor 2-deoxy-D-glucose enhances the efficacy of etoposide in ehrlich ascites tumor-bearing mice. Cancer Biol Ther 2005; 4: 87-94.

35. Chifenti B, Morelli M, Zavaglia M, Coviello DA, Guerneri S, Santucci A et al. Establishment and characterization of 4 new human pancreatic cancer cell lines: evidences of different tumor phenotypes. Pancreas 2009; 38: 184-196.

36. Colak A, Antar V, Karaoglan A, Akdemir O, Sahan E, Celik O et al. Q-VD-OPh, a pancaspase inhibitor, reduces trauma-induced apoptosis and improves the recovery of hind-limb function in rats after spinal cord injury. Neurocirugia (Astur) 2009; 20: 533-540 discussion 540 .

37. Blum R, Jacob-Hirsch J, Rechavi G, Kloog Y. Suppression of survivin expression in glioblastoma cells by the Ras inhibitor farnesylthiosalicylic acid promotes caspasedependent apoptosis. Mol Cancer Ther 2006; 5: 2337-2347.

38. Blum R, Elkon R, Yaari S, Zundelevich A, Jacob-Hirsch J, Rechavi G et al. Gene expression signature of human cancer cell lines treated with the Ras inhibitor salirasib (S-farnesylthiosalicylic acid). Cancer Res 2007; 67: 3320-3328.

39. Ramirez-Peinado S, Alcazar-Limones F, Lagares-Tena L, El Mjiyad N, Caro-Maldonado A, Tirado $\mathrm{OM}$ et al. 2-deoxyglucose induces Noxa-dependent apoptosis in alveolar rhabdomyosarcoma. Cancer Res 2011; 71: 6796-6806.

Cell Death and Disease is an open-access journal published by Nature Publishing Group. This work is licensed under the Creative Commons Attribution-Noncommercial-No Derivative Works 3.0 Unported License. To view a copy of this license, visit http://creativecommons.org/licenses/by-nc-nd/3.0/ 